\title{
Locoregional disease control after external beam radiotherapy in 91 patients with differentiated thyroid carcinoma and pT4 tumor stage - a single institution experience
}

\author{
Nikola Besic ${ }^{1}$, Marta Dremelj², Gasper Pilko ${ }^{1}$ \\ ${ }^{1}$ Department of Surgical Oncology, Institute of Oncology Ljubljana, Ljubljana, Slovenia \\ ${ }^{2}$ Department of Radiotherapy, Institute of Oncology Ljubljana, Ljubljana, Slovenia
}

Radiol Oncol 2018; 52(4): 453-460.

Received 04 May 2018

Accepted 23 May 2018

Correspondence to: Prof. Nikola Bešić, M.D., Ph.D., Department of Surgical Oncology, Institute of Oncology Ljubljana, Zaloška 2, SI-1000 Ljubljana, Slovenia. Phone:+386 15879 994; Fax: +386 15879 998; E-mail: nbesic@onko-i.si

Disclosure: No potential conflict of interest were disclosed.

Background. Locoregional recurrence is common in patients with locally advanced differentiated thyroid carcinoma (DTC). Our aim was to find out the rate of locoregional control of the disease after external beam radiotherapy (EBRT) of the neck and mediastinum in patients with DTC and PT4 tumor.

Patients and methods. Altogether 91 patients (47 males, 44 females, median age 61 years) with DTC had EBRT of the neck and mediastinum as part of the multimodal treatment of pT4 tumor ( 63 cases pT4a, 28 cases pT4b) from the year 1973 to 2015. Data on clinical factors, histopathology and recurrence were collected. Disease-free, diseasespecific and overall survival was calculated.

Results. Median tumor size was $5 \mathrm{~cm}$ (range 1-30 cm). Out of 91 patients, 23 had distant and 38 regional metastases. A total or near-total thyroidectomy, lobectomy, subtotal thyroidectomy and lymph node dissection was performed in $70 \%, 14 \%, 2 \%$ and $30 \%$ of cases, respectively. Thirteen percent of patients were not treated with surgery. All patients had EBRT and 39 had chemotherapy. Radioiodine (RAl) ablation of thyroid remnant and RAl therapy was applied in $90 \%$ and $40 \%$ of cases, respectively. Recurrence was diagnosed in 29/64 patients without a persistent disease: locoregional and distant in 16 and 13 cases, respectively. Five-year and ten-year disease-free survival rate was $64 \%$ and $48 \%$, respectively.

Conclusions. The majority of patients with DTC and PT4 tumors who were treated with EBRT of the neck and mediastinum region as part of multimodal treatment have long-lasting locoregional control of the disease.

Key words: thyroid carcinoma; radiotherapy; survival; pathology; locoregional recurrence

\section{Introduction}

The majority of patients with papillary and follicular carcinoma, i.e., differentiated thyroid carcinoma (DTC), are successfully treated by a combination of surgery, radioiodine and L-thyroxin therapy. ${ }^{1}$ However, 5 to $20 \%$ of patients with differentiated thyroid carcinomas have local or regional recurrences. ${ }^{1}$ Local or lymph nodes relapses are associated with worse survival of patients with differentiated thyroid carcinoma, so every effort should be made to minimize local or regional relapse. ${ }^{2}$ Apart from surgery, postoperative radioactive iodine (RAI) and external beam radiotherapy (EBRT) can reduce locoregional relapse, especially in patients with the postoperative locoregional residual dis-

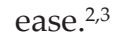

Only a limited number of series have been published about results of EBRT treatment in patients with locally advanced differentiated thyroid carcinoma. ${ }^{2,-14}$ Management of about $10 \%$ of patients with the locally advanced disease remains contro- 
versial. ${ }^{12}$ Our aim was to find out the rate of locoregional control of the disease after external beam radiotherapy (EBRT) of the neck and mediastinum in patients with DTC and pT4 tumor treated in a comprehensive tertiary cancer center.

\section{Patients and methods}

The Cancer Registry of the Republic of Slovenia is one of the oldest population-based registries in Europe. It was founded in 1950 at the Institute of Oncology in Ljubljana (IOL) as a special service for collecting and processing data on cancer incidence and cancer patients' survival. According to data from the Cancer Registry 3,566 patients were treated for thyroid carcinoma in Republic of Slovenia during the period from 1973 to $2015 .{ }^{15}$ All patients with thyroid carcinoma from our country are treated at IOL, which is the only comprehensive cancer center with the only radiotherapy facility equipped with the equipment and systems to plan, carry out and ensure the quality of ionizing radiation treatment in Slovenia. Among 3146 patents with DTC 91 patients (47 males, 44 females; median age 61 years, range 19-87 years) with DTC had EBRT of the neck and mediastinum as part of the multimodal treatment of DTC and pT4 tumor from the year 1973 to 2015 . The mean tumor diameter was $6.3 \mathrm{~cm}$ (range 1-30 cm). Follicular, papillary and Hürthle cell carcinoma was diagnosed in 27, 53 and 11 cases, respectively.

Approval for the study was obtained from The Medical Ethics Committee of the Republic of Slovenia, the Protocol Review Board (MZ 32/12/11), and Protocol Review Board Ethics Committee of the Institute of Oncology (KSOPKR/18/11/10). The trial was retrospectively registered in ISRCTN registry - Study number 34641. The study was conducted in accordance with the ethical standards laid down in an appropriate version of the 1964 Declaration of Helsinki. The study was conducted with the understanding and the consent of the subjects. All patients are asked upon their first admission to the IOL or during a follow-up visit to give consent for the study of her/his chart and/or bioptic material for scientific purposes. Since the Institutional Review Board of the IOL approved this specific study, the patients were not asked to give their written consent for this specific study.

All histological slides of our patients with DTC were reexamined by the pathologist experienced in thyroid pathomorphology. DTC was diagnosed on the histological criteria defined by Rosai and
LiVolsi. ${ }^{16,17}$ The diagnosis of malignancy of follicular and Hürthle cell carcinoma was based on histologic evidence of transcapsular and/or vascular invasion, extrathyroidal local tissue invasion by the primary tumor, or presence of nodal or distant metastasis. An oncocyte was characterized by the presence of acidophilic, granular cytoplasm and hyperchromatic or vesicular nuclei with a large nucleolus. Only lesions demonstrating more than $75 \%$ of follicular cells with oncocytic characteristics were classified as Hürthle cell carcinoma. All patients with Hürthle cell neoplasms with cells containing typical nuclear features of papillary carcinoma were classified as Hürthle cell papillary thyroid carcinoma.

The charts were reviewed, and data on patients' age, gender, disease history, the extent of disease, histomorphological characteristics, modalities of therapy, outcome, and survival were collected. Demographic and clinical characteristics, treatment, and patient outcome are presented in Table 1 and Table 2. Disease-specific survival, overall survival, disease-free survival, locoregional relapse free survival, disease-specific survival according to T4a and T4b tumor stage and disease-specific survival according to distant metastases are presented in Figures 1 to 4 . According to TNM classification, T4a tumors have gross extra-thyroid extension in soft tissue, larynx, trachea, esophagus or recurrent laryngeal nerve, while $\mathrm{T} 4 \mathrm{~b}$ tumors have gross extra-thyroid extension in prevertebral fascia or encasing the carotid artery or gross mediastinal vessels. ${ }^{18}$

The tumor stage, presence of regional and/or distant metastases, as well as a residual tumor after surgery were assessed by the TNM clinical classification according to the 7th edition of UICC criteria from 2009.18 The initial diagnostic work-up in all patients with DTC included ultrasound (US) of the neck region and a chest $X$-ray. The criteria for disease-free survival after thyroid surgery and RAI ablation of thyroid remnant were: thyroglobulin $(\mathrm{Tg})$ levels of less than $1 \mathrm{ng} / \mathrm{mL}$, negative whole-body RAI scans, and the exclusion of cervical lymph node metastases by the US. Additional diagnostic workup was conducted whenever the medical history, physical examination, laboratory findings, and/or radioiodine (RAI) uptake indicated recurrence and/ or distant metastases. It comprised X-ray investigation, US investigation, computed tomography, magnetic resonance imaging, bone scintigraphy, scintigraphy with MIBI and/or PET-CT.

All patients with DTC had a follow-up exam at our Institute at least once per year, while for all 
patients with a recurrence or distant metastases follow-ups were performed at least twice per year. This consisted of meticulously taking their medical history, a physical examination, and determining serum $\mathrm{Tg}$ concentration. Imaging was also conducted whenever $\mathrm{Tg}$ concentration increased, or clinical symptoms or signs suggested that the disease had recurred and/or progressed.

\section{Treatment}

Surgical treatment of primary tumor, locoregional recurrences, and distant metastases was not uniform during the 42-year period; neither was the proportion of patients treated by RAI ablation of thyroid remnant tissue, EBRT, chemotherapy and/ or RAI therapy.

Surgery is the mainstay of DTC treatment. Among surgically treated patients, $84 \%$ had primary surgery at the IOL and $16 \%$ elsewhere, while all other specific therapies (surgery for locoregional recurrence, RAI, EBRT and/or chemotherapy) were applied and follow-ups were conducted for all patients at the IOL. After surgery, all patients received a thyroid hormone replacement titrated to suppress thyroid stimulating hormone (TSH) production.

The data on the type of surgery for primary tumors are listed in Table 1 and Table 2. Total or near-total thyroidectomy is considered an appropriate surgical procedure for DTC. Generally, after an inadequate surgical procedure, a completion thyroidectomy is performed. However, in our study group, this was performed in only five of 21 patients with inadequate surgery. It was not performed if the patient had a recurrent nerve injury $(\mathrm{N}=7)$, preferred treatment with RAI $(\mathrm{N}=5)$, if the patient was very old or with severe comorbidities $(\mathrm{N}=3)$ or the patient refused another surgical procedure $(\mathrm{N}=1)$. Metastases in regional lymph nodes were treated surgically by functional radical neck dissection in 29 patients as part of primary treatment. Twelve of our patients had surgically unresectable tumor because it encasted the carotid artery or gross mediastinal vessels, so they were treated by other treatment modalities.

The initial treatment in 13 patients with a locally advanced tumor was neoadjuvant chemotherapy; of these three patients received concomitant EBRT. Before surgery two patients were treated with preoperative EBRT only. Tumor size decreased in all of these patients, and in five of 15 patients, the largest tumor diameter decreased by more than $30 \%$. Neoadjuvant chemotherapy consisted of long infu-
TABLE 1. Clinical data and outcome of 91 patients with PT4 tumor stage

\begin{tabular}{|c|c|c|}
\hline Characteristic & Subgroup & $\begin{array}{l}\text { Number of } \\
\text { patients }\end{array}$ \\
\hline Gender & $\begin{array}{l}\text { Female } \\
\text { Male }\end{array}$ & $\begin{array}{l}44 \\
47\end{array}$ \\
\hline Patient's age & $\begin{array}{l}44 \text { or less } \\
45 \text { or more }\end{array}$ & $\begin{array}{l}15 \\
76\end{array}$ \\
\hline Tumor diameter $(\mathrm{cm})$ & $\begin{array}{l}1-9 \\
9.1-30\end{array}$ & $\begin{array}{l}75 \\
16\end{array}$ \\
\hline T stage & $\begin{array}{l}\text { pT4a } \\
\text { pT4b }\end{array}$ & $\begin{array}{l}63 \\
28\end{array}$ \\
\hline Lymph nodes metastases & $\begin{array}{l}\text { No } \\
\text { Yes }\end{array}$ & $\begin{array}{l}53 \\
38\end{array}$ \\
\hline Distant metastases & $\begin{array}{l}\text { No } \\
\text { Yes }\end{array}$ & $\begin{array}{l}68 \\
23\end{array}$ \\
\hline $\begin{array}{l}\text { Residual tumor after } \\
\text { surgery }\end{array}$ & $\begin{array}{c}\text { RO } \\
\text { R1 } \\
\text { R2 } \\
\text { No surgery }\end{array}$ & $\begin{array}{c}27 \\
47 \\
5 \\
12\end{array}$ \\
\hline Recurrence & $\begin{array}{c}\text { No } \\
\text { Yes-distant } \\
\text { Yes-locoregional } \\
\text { Disease present permanently }\end{array}$ & $\begin{array}{l}39 \\
13 \\
16 \\
23\end{array}$ \\
\hline Outcome & $\begin{array}{c}\text { Alive } \\
\text { Dead of distant disease } \\
\text { Dead of local disease } \\
\text { Dead of local and distant disease } \\
\text { Dead of other causes } \\
\text { Lost from follow-up }\end{array}$ & $\begin{array}{c}44 \\
29 \\
1 \\
4 \\
10 \\
3\end{array}$ \\
\hline
\end{tabular}

TABLE 2. Treatment of patients

\begin{tabular}{|lcc}
\hline Characteristic & Subgroup & $\begin{array}{c}\text { Number of } \\
\text { Patients }\end{array}$ \\
\hline Surgery of the thyroid & Lobectomy or less & 15 \\
& Total or near-total thyroidectomy & 64 \\
Without surgery & 12 \\
Neck dissection & No & 64 \\
Radioiodine ablation & Yes & 27 \\
& No & 9 \\
Therapy with radioiodine & No & 82 \\
\hline Radiotherapy - & Yes & 54 \\
Neck region & No & 37 \\
Chemotherapy & Yes & 0 \\
& No & 91 \\
\hline \multirow{2}{*}{ Tyrosine kinase inhibitors } & Yes & 52 \\
\hline
\end{tabular}

sion of low dose vinblastine $(2 \mathrm{mg}$ in 12 -hour or 24-hour) in five patients and vinblastine and doxorubicin (20 $\mathrm{mg}$ in 2-hour infusion) in three patients. Neoadjuvant chemotherapy consisted of vinblastine and cis-platinum in one patient, of vinblastine, doxorubicin, and mitoxantrone in one patient, while in three patients more than three cytostatic drugs were used. Altogether 39 patients were treated with chemotherapy. Tyrosine kinase inhibitors were used in two patients included in this study.

RAI was used to ablate thyroid remnant tissue in $82(90 \%)$ patients. Altogether 20 of 27 patients 
(74\%) with non-adequate surgery had the ablation of thyroid remnant tissue. The ablation dose was 3.4-4.8 GBq (92-129 mCi) of RAI. RAI was also used for the treatment of distant metastases and/ or inoperable locoregional disease with an empiric dose of 3.7-7.4 GBq (100-200 mCi). Patients with distant metastases underwent total thyroidectomy before treatment with RAI to increase the uptake of iodine in metastases and to speed up the treatment whenever possible. Before 2002, serum concentration of TSH of $>30 \mathrm{mU} / \mathrm{L}$ was achieved by a 4-6 week withdrawal of L-thyroxin suppression therapy. Since 2002, recombinant human TSH (rhTSH)aided RAI therapy has been used in altogether 13 patients who were elderly and had concomitant diseases, had a history of severe hypothyroid or compressive symptoms and/or evidence of tumor progression during thyroid hormone withdrawal. ${ }^{19}$

All 91 patients included in this report had EBRT to the neck and superior mediastinum. Six patients were treated preoperatively with EBRT, four of them with concomitant chemotherapy and two with EBRT only. Even 63 (69\%) of our patents were irradiated using antero-posterior opposed fields in the supine position with an extended neck. The target volume extended from mastoid tips cranially to tracheal bifurcation caudally and to the coracoids laterally, to cover neck lymph nodes regions I-VI, thyroid bed, and superior mediastinal lymph nodes. The two beams, using Cobalt unit or $6 \mathrm{MV}$ photon beams, were weighted anteriorily; dose from the anterior field was 1.2 Gray (Gy) and from posterior field 0.6 Gy per fraction, 5 fractions per week. The apices of the lungs were shielded; after the received dose of 39.6 Gy. The spinal cord was also shielded from the posterior field, so the dose to the spinal cord was kept bellow $45 \mathrm{~Gy}$. The prescribed dose to the midplane in the vast majority of postoperative patients was $50.4 \mathrm{~Gy}$. A boost dose of 6-16 Gy with 1.8-2 Gy per fraction was delivered to some patients with the macroscopic remnant of disease using photon beams or electron beams avoiding spinal cord. Eight patients were irradiated by 3D technique (TD 50.4-70 Gy, median 63.5 Gy) and 20 patients by intensity-modulated radiotherapy technique (IMRT) to the whole neck and mediastinum (TD 56-70 Gy, median 64 Gy), while only one patient had only thyroid bed irradiation. The five of six patients treated with EBRT before surgery received 36 Gy before surgery and 14 Gy after surgery, while one patient treated with EBRT before surgery received 50 Gy with IMRT technique.

EBRT toxicity was difficult to estimate since in some patients it was inadequately documented.
Ten patients had radiotherapy interrupted for one to three weeks because of acute mucositis or dermatitis, but eventually finished the prescribed therapy. One patient stopped postoperative radiotherapy at 16 Gy due to reported side effects, received further radioiodine therapy and progressed two years later at a distant site. Among 28 patients treated with IMRT or 3D technique Grade $3 \mathrm{mu}-$ cositis or Grade 3 dermatitis was reported in 10 patients. Furthermore, a weight loss above $5 \mathrm{~kg}$ was detected in eight patients who also needed hospitalization for parenteral therapy, in two of them a nasogastric tube was placed, while in one patient a tracheostomy was done. Of the late effects, a grade 2-3 neck fibrosis was documented in two patients and Lhermitte's sign in one patient. One patient had tracheal stricture after partial resection of the trachea which was resolved with laser therapy, and the patient is alive with very slowly progressing regional and distant disease recurrence 17 years after the beginning of treatment.

\section{Survival}

Disease-specific survival was defined as the period from primary treatment (surgery, chemotherapy or EBRT) to death or the last follow-up. Disease-free survival was defined as the period from primary surgical treatment to the radiologic or morphologic diagnosis of locoregional recurrence, distant metastases or the last follow-up. Locoregional relapse free survival was analyzed in patients without gross residual tumor. Locoregional relapse free survival was defined as the period from primary surgical treatment to the radiologic or morphologic diagnosis of locoregional recurrence or the last follow-up.

Overall survival was defined from the period of primary treatment to death of any cause or the last follow-up. The median duration of follow-up was 5.1 years (range 0.5-25.7 years).

\section{Statistical analysis}

The Student t-test or the Mann-Whitney U-test was used according to data distribution. The association between categorical variables was tested by the chi-square test or Fisher's exact test, as appropriate. All comparisons were two-sided and a p-value $<0.05$ was considered statistically significant. The statistical correlation between possible prognostic factors and cause-specific survival was analyzed by chi-square test and log-rank analysis. The estimated survival curves were calculated ac- 


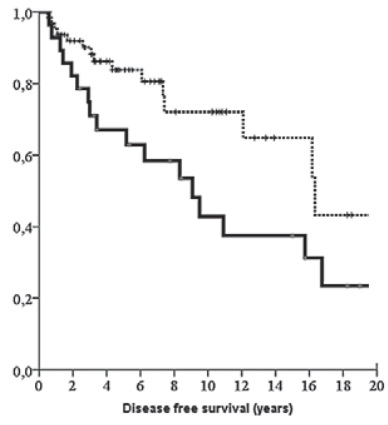

FIGURE 1. Disease-specific survival according to T4a and T4b tumor stage $(p=0.07)$ (bold line, T4b; dotted line, T4a).

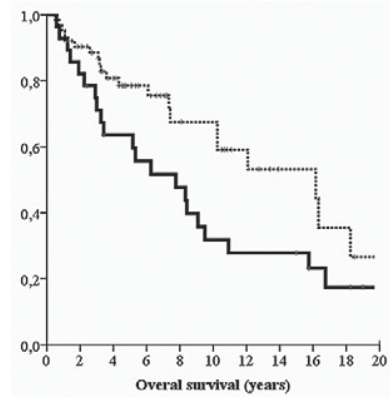

FIGURE 2. Overall survival according to T4a and T4b tumor stage $(p=0.08)$ (bold line, T4b; dotted line, T4a).

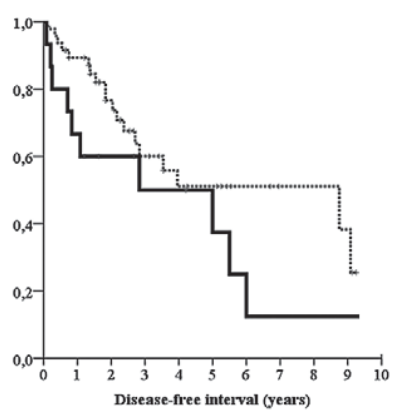

FIGURE 3. Disease-free survival according to T4a and T4b tumor stage in 64 patients without persistent disease $(p=0.122)$ (bold line, T4b; dotted line, T4a).

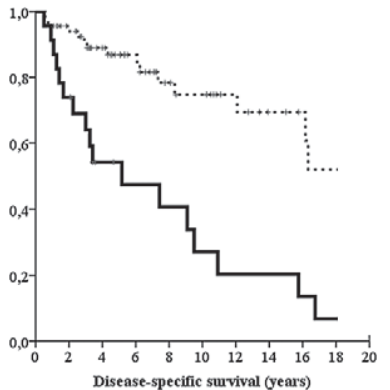

FIGURE 4. Patients without distant metastases had longer disease-specific survival than those without distant metastases ( $p<0.001$ ) (bold line, with metastases; dotted line, without metastases). cording to the Kaplan-Meier method. Multivariate statistical analysis was not performed because of the small number of patients. The statistical package PASW 18 (SPSS Inc., Chicago, IL, USA) was used for the analysis.

\section{Results}

Follicular, papillary and Hürthle cell carcinoma was diagnosed in 27, 53 and 11 cases, respectively. Median tumor size was $5 \mathrm{~cm}$ (range 1-30 cm). Of 91 patients, 23 had distant and 38 regional metastases (Table 1). A total or near-total thyroidectomy, lobectomy, subtotal thyroidectomy and lymph node dissection was performed in $70 \%$, $14 \%, 2 \%$ and $30 \%$ of cases, respectively. $13 \%$ of patients were not treated with surgery. All patients had EBRT and 39 had chemotherapy. Radioiodine (RAI) ablation of remnant thyroid tissue and RAI therapy was done in $90 \%$ and $40 \%$ of cases, respectively (Table 2).

\section{Locoregional control of disease}

The follow-up period was 6 to 308 (median 61) months. Recurrence was diagnosed in 29/68 (43\%) patients without a persistent disease: locoregional and distant in 16 and 13 cases, respectively. Fiveyear and 10-year recurrence-free survival was in $64 \%$ and $48 \%$, respectively. Locoregional control of disease was achieved in 49/64 (77\%) of patients.

Locoregional recurrence or progression of residual locoregional carcinoma was detected in $21 \%$ of patients after $\mathrm{R} 1$ resection, $30 \%$ after $\mathrm{R} 2$ resection and $33.3 \%$ of patients with unresectable disease. Of the 18 patients who had gross residual neck disease without distant metastases after a surgical procedure, 7 demonstrated complete response, 6 had locoregional progression (after 6, 17, 26, 49, 68 and 144 months) and 5 only distant progression of the disease.

\section{Survival}

By the end of the study, 44 patients were still alive (28 with no evidence of disease, 16 alive with the disease), 3 were lost to follow-up, 34 patients died of thyroid carcinoma (29 of distant metastases, 1 of locoregional disease, and 4 of locoregional disease and distant metastases), while 10 died of causes unrelated to the primary disease.

The median survival of follicular, Hürthle cell and papillary carcinoma was 62, 131 and 240 months, respectively. Estimated locoregional relapse-free survival, disease-specific survival, and overall survival at 4 years were $71 \%, 79 \%$, and $75 \%$, respectively. Five-year and 10-year cancerspecific survival in patients with $\mathrm{T} 4 \mathrm{a}$ and $\mathrm{T} 4 \mathrm{~b}$ tumors was $77 \%$ and $60 \%$ (Figure 1). Five-year and 10-year overall survival in patients with T4a and T4b tumors was $72 \%$ and $49 \%$ (Figure 2). Five-year and 10-year disease-free survival in patients with $\mathrm{T} 4 \mathrm{a}$ and $\mathrm{T} 4 \mathrm{~b}$ tumors was $64 \%$ and $48 \%$ (Figure 3 ). Figure 4 shows that patients without distant metastases had longer disease-specific survival than those with distant metastases $(\mathrm{p}<0.001)$.

Of the 18 patients who had gross residual neck disease without distant metastases after surgical procedure, 7 are alive $(29,37,40,57,97,153$ and 228 months) and 4 died of other causes $(39,64,123$ and 219 months), while 6 died of distant metasta- 
ses after 2, 6, 17, 26, 31 and 52 months and one is lost from follow-up.

Ten of 12 patients who were not surgically treated are dead, and 8 of 10 died of DTC. They were alive for $9,11,17,17,27,39,62,114$ and 194 months. Locoregional control of disease was achieved in 5 of 8 patients. Five patients died of distant metastases, one of the locally progressive tumor, while two patients died of distant and locoregional disease.

\section{Discussion}

In a cohort of 1,297 patients, Chow et al. reported that 100 patients died because of thyroid cancer. ${ }^{9}$ The uncontrolled locoregional disease was the cause of death in as many as 59/100 patients. ${ }^{9}$ Locoregional recurrence of DTC is a heavy burden for individual patients, as well as for the health care system. Locoregional recurrence in the majority of cases necessitates reoperation, with significant consequential morbidity and costs. ${ }^{12}$ Furthermore, recurrences located in the thyroid bed with soft tissue infiltration are less likely to be iodine avid so that they can be rarely cured with radioiodine. ${ }^{20}$ Moreover, not all patients with recurrence can be successfully saved. Therefore, prevention of locoregional recurrence is very reasonable. Our aim was to find out the rate of locoregional control of the disease after EBRT of the neck and mediastinum in patients with DTC and pT4 tumor treated in a tertiary cancer comprehensive center.

Adjuvant therapy is administered in addition to the primary, main, or initial therapy to maximize its effectiveness. Adjuvant systemic therapy and radiotherapy are often applied following surgery for many types of cancer, including colon cancer, lung cancer, pancreatic cancer, breast cancer, prostate cancer, and some gynecological cancers. ${ }^{21}$ In patients with DTC, adjuvant therapy and therapy for the residual disease may include chemotherapy, EBRT, radioiodine treatment and/or suppressive L-thyroxin therapy. 3,4,22,23

We believe that chemotherapy may be an effective adjuvant treatment in patients with high risk of recurrence, so it was used in as many as $43 \%$ of our patients treated at IOL. Interestingly, this mode of therapy was seldom used in other centers. Schwartz et al. from MD Anderson Cancer Center reported that chemotherapy was used in only 6 of 126 patients (5\%) who were treated with EBRT. ${ }^{12}$

Adjuvant EBRT is another rarely used treatment in locally advanced DTC disease to prevent a locoregional recurrence. So only a limited number of series have been published reporting the results of EBRT treatment in patients with locally advanced DTC. 2,4-14 To our knowledge, only one randomized study about ERBT is reported in the literature. ${ }^{13}$ The Multicenter Study on Differentiated Thyroid Cancer (MSDS) was planned as a prospective multicenter trial on the benefit of adjuvant EBRT in patients with DTC and pT4, with or without lymph node metastases and no known distant metastases. ${ }^{13}$ However, only 45 of planned 311 patients were randomized. Not surprisingly, only a weak benefit of EBRT regarding local control was observed that did not reach statistical significance. ${ }^{13}$

Gross, the unresectable residual disease is accepted as an indication for adjuvant radiotherapy in the 2015 American Thyroid Association Management Guidelines for Adult Patients with Thyroid Nodules and Differentiated Thyroid Cancer. ${ }^{23}$ Schwartz et al. reported that a total of 15 patients received EBRT for gross unresectable or residual disease. ${ }^{12}$ After EBRT of the 15 patients, 4 demonstrated a complete response, 3 had a partial response, 6 had stable disease, and 2 progressed through treatment. ${ }^{12}$ Our multimodal treatment resulted in better locoregional control and longer survival in 8 of 12 (67\%) patients who were not surgically treated because of unresectable disease. Of the 18 patients who had gross residual disease after the surgical procedure and were without distant metastases, 7 demonstrated a complete response, 5 had only distant progression, while 6 had locoregional progression after 6 , 17, 26, 49, 68 and 144 months.

EBRT in the neck region is a very difficult task because of its concave shape. ${ }^{12}$ An additional difficulty is the close proximity of the thyroid bed to critical normal structures, which makes it challenging to deliver high doses. ${ }^{12}$ The proximity of the esophagus, trachea, larynx, lungs, spinal cord, and, in cases with the disease in cervical regions I and II also parotid glands, results in considerable toxicity after conventional radiotherapy. ${ }^{12}$ The majority of patients $(69 \%)$ from our study were treated with conventional two-field EBRT. This dose was effective in preventing locoregional recurrence or progression of residual tumor in 49/65 (75\%) patients: $80 \%$ after $\mathrm{R} 1$ resection and $70 \%$ after $\mathrm{R} 2$ resection. None of our patients treated with two-field EBRT needed hospitalization due to side effects or placement of a nasogastric feeding tube. Mild or moderate dysphagia or xerostomia was present in some patients, while Lhermitte's sign was reported in only one patient. A probable explanation for this is a lower dose (median 50.4 Gy) which was used in our patients treated with conventional EBRT. 
However, one should take into account the retrospective nature of our study, so not all side effects were documented and were probably under-reported.

Rosenbluth et al. from Memorial Sloan-Kettering reported the results of a pilot study about IMRT used in 20 patients with differentiated or medullary thyroid cancer. ${ }^{8}$ They reported Grade 3 mucositis in 7 patients, Grade 3 pharyngitis in 3 patients, Grade 3 acute laryngeal toxicity in 2 patients, and Grade 3 skin toxicity in 2 patients. Eleven of their patients received percutaneous endoscopic gastrostomy. However, acute toxicity was mostly self-limited. ${ }^{8}$ In our 20 patients treated with IMRT technique a Grade 3 mucositis or Grade 3 dermatitis was reported in 7 patients. Furthermore, weight loss larger than $5 \mathrm{~kg}$ was detected in six patients who also needed hospitalization for parenteral therapy. In one of our patient's tracheostomy was done. Lower proportion of side effects in our patients in comparison to patients from Memorial Sloan-Kettering may be explained by a lower dose in our patients (median $64 \mathrm{~Gy}$ versus $66 \mathrm{~Gy}$, respectively). On the other hand, the larger volumes of irradiated tissue come at the cost of higher toxicity, so it is important that the volume at risk of relapse is carefully considered. ${ }^{24}$ Brierley reported that they frequently use a more limited volume and the locoregional relapse rate was only $7 \%$ in selected patients with extrathyroidal extension and no gross residual disease. He concluded that more information is required to ascertain which patients require extended field EBRT, as well as the risk of higher acute and late toxicities. ${ }^{24}$

The limitation of our study is that the results of treatment and survival from a single institution are reported. Another limitation is that not all our patients were treated uniformly. During a 42-year time frame, they were treated by many surgeons and oncologists. Our study is observational and not randomized, thus it is not possible to draw conclusions about the impact of a treatment's specific modality on recurrence rates or patients' survival. Due to the retrospective nature of our study side effects of EBRT and IMRT were not completely documented and are probably under-reported.

Our view is that all patients with non-favorable prognoses and/or a high risk of structural disease recurrence should be treated by a multidisciplinary team consisting of a surgeon, a nuclear medicine specialist, a radiotherapist and a medical oncologist. Appropriate surgical procedures consisting of a total thyroidectomy and lymph node dissection of affected compartments should be followed by a radioiodine ablation of remnant thyroid tissue whenever possible. Our results prove that longterm survival can also be obtained in patients with locoregionally advanced DTC and a distant metastatic disease if they are treated multidisciplinary. Chemotherapy before surgical procedures may be effective to decrease the size of the primary tumor in DTC. ${ }^{25-27}$ Concomitant chemotherapy with EBRT in locoregionally advanced DTC can result in complete and very long locoregional control. ${ }^{4}$ Failure to obtain a locoregional control of disease may cause a dismal course of thyroid carcinoma. ${ }^{9,28}$ Since residual tumors after thyroid surgery are an independent prognostic factor for disease-specific as well as disease-free survival, an effective locoregional therapy is mandatory. Our view is that the use of adjuvant EBRT can postpone or prevent treatment with kinase inhibitors which are very expensive and have many very unpleasant and also serious side effects. On the other hand, also IMRT technique of EBRT has serious acute side effects. However, in the majority of patients, acute side effects last only about one month and are self-limiting. Furthermore, the late toxicity of EBRT is rare. Therefore, EBRT should be used in cases of residual tumors and as adjuvant therapy in patients with a high risk of structural disease recurrence.

\section{Conclusions}

The majority of patients with DTC and pT4 tumors who were treated with EBRT of the neck and mediastinum region as part of multimodal treatment have long-lasting locoregional control of the disease. Locoregional recurrence or progression of residual locoregional carcinoma was detected in $21 \%$ of patients after $\mathrm{R} 1$ resection, $30 \%$ after $\mathrm{R} 2$ resection and $33.3 \%$ of patients with unresectable disease.

\section{Acknowledgements}

The paper was supported by a research program, P3-0289, by the Ministry of Higher Education, Science and Sport of Slovenia.

\section{References}

1. Schlumberger MJ. Papillary and follicular thyroid carcinoma. N Engl J Med 1998; 338: 297-306. doi: 10.1056/NEJM199801293380506

2. Chow SM, Law SCK, Mendenhall WM, Au SK, Chan PT, Leung TW, et al. Papillary thyroid carcinoma: prognostic factors and the role of radioiodine and external radiotherapy. Int J Radiat Oncol Biol Phys 2002; 52: 784-95. doi: org/10.1016/S0360-3016(01)02686-4 
3. Brierley J, Tsang R, Panzarella T, Bana N. Prognostic factors and the effect of treatment with radioactive iodine and external beam radiation on patients with differentiated thyroid cancer seen at a single institution over 40 years. Clin Endocrinol (Oxf) 2005; 63: 418-27. doi: 10.1111/j.1365$2265.2005 .02358 x$

4. Kim JH, Leeper RD. Treatment of locally advanced thyroid carcinoma with combination doxorubicin and radiation therapy. Cancer 1987; 60: 2372-5.

5. Philips P, Hanzen C, Andry G, Van Houtte P, Früuling J. Postoperative irradiation for thyroid cancer. Eur J Surg Oncol 1993; 19: 399-404.

6. Farahati J, Reiners C, Stuschke M, Müller SP, Stüben G, Sauerwein W, Sack H. Differentiated thyroid cancer. Impact of adjuvant external radiotherapy in patients with perithyroidal tumor infiltration (stage pT4). Cancer 1996; 77: 172-80. doi: 10.1002/(SICI)1097-0142(19960101)77:1<172::AIDCNCR28>3.0.CO;2-1

7. Tsang RW, Brierley JD, Simpson WJ, Panzarella T, Gospodarowicz MK Sutcliffe SB. The effects of surgery, radioiodine, and external radiation therapy on the clinical outcome of patients with differentiated thyroid carcinoma. Cancer 1998; 82: 375-88.

8. Rosenbluth BD, Serrano V, Happersett L, Shaha AR, Tuttle RM, Narayana A et al. Intensity-modulated radiation therapy for the treatment of nonanaplastic thyroid cancer. Int J Radiat Oncol Biol Phys 2005; 63: 1419-26. doi: 10.1016/j.ijrobp.2005.05.043

9. Chow SM, Yau S, Kwan CK, Poon PC, Law SC. Local and regional control in patients with papillary thyroid carcinoma: specific indications of external radiotherapy and radioactive iodine according to $\mathrm{T}$ and $\mathrm{N}$ categories in AJCC 6th edition. Endocr Relat Cancer 2006; 13: 1159-72. doi: 10.1677/ erc. 1.01320

10. Azrif M, Slevin NJ, Sykes AJ, Swindell R, Yap BK. Patterns of relapse following radiotherapy for differentiated thyroid cancer: implication for target volume delineation. Radiother Oncol 2008; 89: 105-13. doi: 10.1016/j. radonc.2008.05.023

11. Terezakis SA, Lee KS, Ghossein RA, Rivera M, Tuttle RM, Wolden SL et al. Role of external beam radiotherapy in patients with advanced or recurrent nonanaplastic thyroid cancer: Memorial Sloan-Kettering Cancer Center experience. Int J Radiat Oncol Biol Phys 2009; 73: 795-801. doi: 10.1016/j. ijrobp.2008.05.012

12. Schwartz DL, Lobo MJ, Ang KK, Morrison WH, Rosenthal DI, Ahamad A, et al. Postoperative external beam radiotherapy for differentiated thyroid cancer: outcomes and morbidity with conformal treatment. Int J Radiat Oncol Biol Phys 2009; 74: 1083-91. doi: 10.1016/j.jijrobp.2008.09.023

13. Biermann M, Pixberg M, Riemann B, Schuck A, Heinecke A, Schmid KW, et al; MSDS study group. Clinical outcomes of adjuvant external-beam radiotherapy for differentiated thyroid cancer - results after 874 patient-years of follow- up in the MSDS-trial. Nuklearmedizin 2009; 48: 89-98. doi: 10.3413/ nukmed-0221

14. Kim TH, Chung KW, Lee YJ, Park CS, Lee EK, Kim TS, et al. The effect of external beam radiotherapy volume on locoregional control in patients with locoregionally advanced or recurrent nonanaplastic thyroid cancer. Radiat Oncol 2010; 5: 69. doi: 10.1186/1748-717X-5-69

15. Cancer in Slovenia 2014. Ljubljana: Institute of Oncology Ljubljana, Epidemiology and Cancer Registry, Cancer Registry of Republic of Slovenia, 2017. [cited 2018 April 15]. Available from: https://www.onko-i.si/fileadmin/onko/datoteke/dokumenti/RRS/LP_2014.pdf

16. Rosai J. Tumors of thyroid gland. In: Rosai J, Carcangiu ML, DeLellis RA, editors. Atlas of tumor pathology. Washington: Armed Forces Institute of Pathology; 1992. p. 31-50.

17. LiVolsi VA, Baloch ZW. Follicular neoplasms of the thyroid: view, biases, and experiences. Adv Anat Pathol 2004; 11: 279-87. doi: 10.1097/01. pap.0000138143.34505.02

18. Sobin LH, Gospodarowicz MK, Wittekind C. Thyroid gland (ICD-O C73). In: Sobin LH, Gospodarowicz MK, Witekind C, editors. TNM classification of malignant tumours. 7th Edition. New York: Wiley Blackwell; 2009. p. 58-62.

19. Zagar I, Schwarzbartl-Pevec A, Vidergar-Kralj B, Horvat R, Besic N. Recombinant human thyrotropin-aided radioiodine therapy in patients with metastatic differentiated thyroid carcinoma. J Thyroid Res 2012; 670180. doi: $10.1155 / 2012 / 670180$
20. Vassilopoulou-Sellin R, Schultz PN, Haynie TP. Clinical outcome of patients with papillary thyroid carcinoma who have recurrence after initial radioactive iodine therapy. Cancer 1996; 78: 493-501. doi: 10.1002/(SICI)10970142(19960801)78:3<493::AID-CNCR17>3.0.CO;2-U

21. Feig BW, Ching DC editors. The MD Anderson surgical oncology handbook. Philadelphia: Lippincott Williams \& Wilkins; 2012.

22. Santini F, Bottici V, Elisei R, Montanelli L, Mazzeo S, Basolo F, et al. Cytotoxic effects of carboplatinum and epirubicin in the setting of an elevated serum thyrotropin for advanced poorly-differentiated thyroid cancer. J Clin Endocrinol Metab 2002; 87: 4160-5. doi: 10.1210/jc.2001-011151

23. Haugen BR, Alexander EK, Bible KC, Doherty GM, Mandel SJ, Nikiforov YE, et al. 2015 American Thyroid Association Management Guidelines for Adult Patients with Thyroid Nodules and Differentiated Thyroid Cancer. Thyroid 2016; 26: 1-133. doi: 10.1089/thy.2015.0020

24. Brierley JD. Update on external beam radiation therapy in thyroid cancer. $J$ Clin Endocrinol Metab 2011; 96: 2289-95. doi: 10.1210/jc.2011-1109

25. Besic N, Auersperg M, Gazic B, Dremelj M, Zagar I. Neoadjuvant chemotherapy in 29 patients with locally advanced follicular or Hürthle cell thyroid carcinoma: a phase 2 study. Thyroid 2012; 22: 131-7. doi: 10.1089/ thy.2011.0243

26. Besic N, Auersperg M, Dremelj M, Vidergar-Kralj B, Gazic B. Neoadjuvant chemotherapy in 16 patients with locally advanced papillary thyroid carcinoma. Thyroid 2013; 23: 178-84. doi: 10.1089/thy.2012.0194

27. Besic N, Dremelj M, Schwartzbartl-Pevec A, Gazic B. Neoadjuvant chemotherapy in 13 patients with locally advanced poorly differentiated thyroid carcinoma based on Turin proposal - a single institution experience. Radiol Oncol 2015; 49: 271-8. doi: 10.1515/raon-2015-0001

28. Vogrin A, Besic H, Besic N, Music MM. Recurrence rate in regional lymph nodes in 737 patients with follicular or Hürthle cell neoplasms. Radiol Oncol 2016; 50: 269-73. doi: 10.1515/raon-2016-0025 